\title{
Fibonacci and Lucas Identities with the Coefficients in Arithmetic Progression
}

\author{
Soumendra Bera \\ Mahishadal Raj College, Vidyasagar University, W.B., India
}

\begin{abstract}
We define an analogous pair of recurrence relations that yield some Fibonacci, Lucas and generalized Fibonacci identities with the coefficients in arithmetic progression. One relation yields same sign identities and the other alternating signs identities. We also show some new results for negative indexed Fibonacci and Lucas Sequences.
\end{abstract}

Keywords: linear recurrence relation; Fibonacci sequence; Lucas identity; generalized Fibonacci sequence, arithmetic progression.

\section{Introduction}

Fibonacci sequence is defined by the linear recurrence relation: $F_{n+1}=F_{n}+F_{n-1}$ for $n \geq 1$ with the initial conditions: $F_{0}=0$ and $F_{1}=1$. Lucas sequence is similar to Fibonacci sequence and defined by the relation: $L_{n+1}=L_{n}+L_{n-1}$ for $n \geq 1$ with the initial conditions: $L_{0}=2$ and $L_{1}=1$. Generalized Fibonacci sequence is defined by the relation: $G_{n+1}=G_{n}+G_{n-1}$ for $n \geq 1$ with the initial conditions: $G_{0}=x$ and $G_{1}=$ $y$ for some $x$ and $y$. Obviously Fibonacci and Lucas sequences are the special cases of generalized Fibonacci sequence with $(0,1)$ and $(2,1)$ as the values of the pair $(x, y)$ respectively.

A generalized Fibonacci formula is given by the following recurrence relation:

$$
\text { For } 0 \leq m \leq n, \quad G_{n+m}=L_{m} G_{n}+(-1)^{m+1} G_{n-m} \text {. }
$$

(1) is known. This is the formula (10a) in 'List of formulae' in the book, Fibonacci and Lucas Numbers, and the Golden Section: Theory and Applications by Steven Vajda; and also the identity 45 in the book, Proofs That Really Count: The Art of Combinatorial Proof by Arthur Benjamin and Jennifer Quinn. Fibonacci and Lucas numbers both satisfy (1). So from (1), we get:

$$
\begin{aligned}
& \text { For } 0 \leq m \leq n, \quad F_{n+m}=L_{m} F_{n}+(-1)^{m+1} F_{n-m} . \\
& \text { For } 0 \leq m \leq n, \quad L_{n+m}=L_{m} L_{n}+(-1)^{m+1} L_{n-m} .
\end{aligned}
$$

We obtain Fibonacci and Lucas identities with the coefficients in arithmetic progression by using (1.1), (1.2) and two recurrence relations which are defined in the next topic.

\section{(a) Recurrence Relation 1}

\section{Pair of Analogous Recurrence Relations And Their Transformations}

For some numbers: $a, b$ and $S_{0}$, we define an $n^{\text {th }}$ order recurrence function $S_{n}$ for $n \geq 1$ by the linear recurrence relation:

From (2),

$$
S_{n}=\sum_{i=0}^{n-1}(a+i b) S_{n-1-i}+(a+n b) .
$$

$$
\begin{aligned}
& S_{n+1} \quad=a S_{n}+(a+b) S_{n-1}+\ldots+(a+n b) S_{0}+\{a+(n+1) b\} . \\
& \quad \quad=a S_{n}+a S_{n-1}+\ldots+\{a+(n-1) b\} S_{0}+(a+n b)+b\left(S_{n-1}+\ldots+S_{0}+1\right) . \\
& \Rightarrow S_{n+1}=a S_{n}+S_{n}+b\left(S_{n-1}+\ldots+S_{0}+1\right) .
\end{aligned}
$$

Similarly

$$
\begin{aligned}
& S_{n+2}=a S_{n+1}+(a+b) S_{n}+\ldots+(a+(n+1) b) S_{0}+(a+(n+2) b) . \\
& \Rightarrow S_{n+2}=a S_{n+1}+S_{n+1}+b\left(S_{n}+\ldots+S_{0}+1\right) .
\end{aligned}
$$

From (2.2) and (2.4), 


$$
\begin{aligned}
& b\left(S_{n-1}+\ldots+S_{0}+1\right)=S_{n+2}-(a+1) S_{n+1}-b S_{n} \\
&= S_{n+1}-(a+1) S_{n} \\
& \Rightarrow S_{n+2}=(a+2) S_{n+1}+(b-a-1) S_{n} .
\end{aligned}
$$

(2.5) is a second order linear recurrence relation and a reduced version of (2.3) with the initial conditions: $S_{1}=$ $a S_{0}+(a+b)$ and $S_{2}=\left(a^{2}+a+b\right) S_{0}+\left(a^{2}+a b+a+2 b\right)$.

\section{(b) Recurrence Relation 2}

For some numbers: $a, b$ and $T_{0}$, we define an $n^{\text {th }}$ order recurrence function $T_{n}$, for $n \geq 1$ by the linear recurrence relation:

$$
T_{n}=\sum_{i=0}^{n-1}(-1)^{i}(a+i b) T_{n-1-i}+(-1)^{n}(a+n b) .
$$

We obtain $(2.1), \ldots$, (2.5) from (2). In like manner we obtain below (3.1), .., (3.5) from (3):

$$
\begin{aligned}
T_{n+1} & =\sum_{i=0}^{n}(-1)^{i}(a+i b) T_{n-i}+(-1)^{n+1}\{a+(n+1) b\} . \\
T_{n+1} & =a T_{n}-T_{n}+b\left\{-T_{n-1}+\ldots+(-1)^{n} T_{0}+(-1)^{n+1}\right\} . \\
T_{n+2} & =\sum_{i=0}^{n+1}(-1)^{i}(a+i b) T_{n+1-i}+(-1)^{n+2}\{a+(n+2) b\} . \\
T_{n+2} & =a T_{n+1}-T_{n+1}+b\left\{-T_{n}+\ldots+(-1)^{n+1} T_{0}+(-1)^{n+2}\right\} . \\
T_{n+2} & =(a-2) T_{n+1}+(a-b-1) T_{n} .
\end{aligned}
$$

(3.5) is a second order linear recurrence relation and the reduced version of (3.3) with the initial conditions: $T_{1}=$ $a T_{0}-(a+b)$; and $T_{2}=\left(a^{2}-a-b\right) T_{0}-\left(a^{2}+a b-a-2 b\right)$.

(c) Transformation of Second Order Linear Recurrence Relation to $n$-th Order Linear Recurrence Relations of Two Kinds

It follows from (2), (2.5), (3) and (3.5) that second order linear relation:

$$
U_{n+2}=x U_{n+1}+y U_{n}
$$

has $n^{\text {th }}$ order versions of two kinds. (4) is (2.5) with the conditions: $x=a+2$ and $y=b-a-1$; and is (3.5) with the conditions: $x=a-2$ and $y=a-b-1$. In other words (i) putting $a=x-2$ and $b=x+y-1$ in (2), we find the same sign $n$-th order version of (4); and (ii) putting $a=x+2$ and $b=x-y+1$ in (2), we find the alternating signs $n$-th order version of (4).

\section{Usual Or Positive Indexed Fibonacci And Lucas Identities With The Coefficients In Arithmetic Progression}

(a) Fibonacci and Lucas Identities from Recurrence Relation 1

(2.5) is analogous with (1).

(i) Putting $a=b=L_{m}-2$ in (2.5), we get:

$$
S_{n+2}=L_{m} S_{n+1}-S_{n} .
$$

(ii) Putting $a=L_{m}-2$ and $b=L_{m}$ in (2.5), we get:

$$
S_{n+2}=L_{m} S_{n+1}+S_{n} .
$$

The values of the pair $(a, b)$ and $L_{m}$ for $m \in(1,2,3,4)$ are listed in Table 1. 
Table 1

\begin{tabular}{|l|l|l|}
\hline $\boldsymbol{m}$ & \multicolumn{1}{|c|}{$\boldsymbol{L}_{\boldsymbol{m}}$} & $(\boldsymbol{a}, \boldsymbol{b})$ \\
\hline 1 & 1 & $(-1,1)$ \\
\hline 2 & 3 & $(1,1)$ \\
\hline 3 & 4 & $(2,4)$ \\
\hline 4 & 7 & $(5,5)$ \\
\hline
\end{tabular}

(5.1) and (5.2) depend on $S_{0}$ also. By trial, it is found that (5.1) and (5.2) represent some particular forms of (1.1) and (1.2) for some values of the pair $\left(m, S_{0}\right)$. Consequently, Recurrence relation 1 (the relation for $S_{n}$ ) or any higher order relation (the relation for $S_{n+k}$ for $k \geq 1$ ) can yield the Fibonacci and Lucas identities with the coefficients in arithmetic progression. But the results are limited. The values of $S_{0}$ for $m \in(1,2,3,4)$, which can generate the desired identities, are listed in Table 2.

\section{Table 2}

\begin{tabular}{|l|l|}
\hline Values of $\boldsymbol{m}$ & Values of $\boldsymbol{S}_{\mathbf{0}}$ \\
\hline 1 & $-4,-3,-2,-1,0,1$ \\
\hline 2 & $-3,-2,-1,0,1,2$ \\
\hline 3 & $-5,-3,-2,-1,0,1,2,3,5$ \\
\hline 4 & $-6,-3,0,3,6,9$ \\
\hline
\end{tabular}

In Row 1 , the values of the pair $\left(m, S_{0}\right)$ are: $(1,-4),(1,-3),(1,-2),(1,-1),(1,0)$, and $(1,1$,$) where the$ common value of $m$ is 1 . All these six pairs can yield the identities of the desired pattern. Similarly in Row 2 , six values of $\left(m, S_{0}\right):(2,-3),(2,-2),(2,-1),(2,0),(2,1)$, and $(2,2)$ can yield six identities; and so on. It is found that some values of the pair: $\left(m, S_{0}\right)$ yield the same identity of desired pattern.

Example: We show the result when $\left(m, S_{0}\right)$ is $(2,-2)$. For $m=2, L_{m}=L_{2}=3$ and $a=b=L_{m}-2=1$; and then for $S_{0}=-2$,

(2) yields: $\quad S_{1}=0=F_{0}$; and $S_{2}=-1=-F_{2}$;

(5.2) yields: $\quad S_{3}=L_{2} S_{2}-S_{1}=-L_{2} F_{2}+F_{0}=-F_{4}$

$$
\begin{aligned}
& S_{4}=L_{2} S_{3}-S_{2}=-L_{2} F_{4}+F_{2}=-F_{6} ; \\
& \ldots \ldots
\end{aligned}
$$

In general,

$$
S_{n+2}=L_{2} S_{n+1}-S_{n}=-L_{2} F_{2 n}+F_{2 n-2}=-F_{2 n+2} \cdot[\text { By (1.1)] }
$$

Then from (2.3), we get the following Fibonacci identity with the coefficients in arithmetic Progression.

$$
\begin{aligned}
& -F_{2 n+2}=-F_{2 n}-2 F_{2 n-2}-\ldots-n F_{0}-(n+1) . \\
& \Rightarrow F_{2 n}=\sum_{i=0}^{n-1} i F_{2(n-i)}+n .
\end{aligned}
$$

We can obtain also the above identity when the value of $\left(m, S_{0}\right)$ is $(2,1)$.

The values of $\left(m, S_{0}\right)$ and the Fibonacci and Lucas identities with respect to Recurrence Relation 1 are listed in Table 3 below. 
Table 3

\begin{tabular}{|c|c|c|c|c|}
\hline Sl. No. & $m$ & $S_{0}$ & $\begin{array}{r}\text { Fibonacci Identities } \\
\end{array}$ & \\
\hline 1. & 1 & $0,-1,-2,-3$ & $F_{n}=\sum_{i=1}^{n}(i-2) F_{n-i}+n$ & (6.1) \\
\hline 2. & 2 & $1,-2$ & $F_{2 n}=\sum_{i=0}^{n-1} i F_{2(n-i)}+n$ & $(6.2)$ \\
\hline 3. & 2 & $0,-1$ & $F_{2 n+1}=\sum_{i=1}^{n} i F_{2(n-i)+1}+1$ & $(6.3)$ \\
\hline 4. & 3 & -3 & $F_{3 n}=2\left\{\sum_{i=1}^{n}(2 i-1) F_{3(n-i)}+n\right\}$ & (6.4) \\
\hline 5. & 3 & $3,-1$ & $F_{3 n+1}=2\left\{\sum_{i=1}^{n}(2 i-1) F_{3(n-i)+1}\right\}+1$ & $(6.5)$ \\
\hline 6. & 3 & $2,-2$ & $F_{3 n+2}=2 \sum_{i=1}^{n}(2 i-1) F_{3(n-i)+2}+2 n+1$ & $(6.6)$ \\
\hline 7. & & & $F_{4 n}=5 \sum_{i=1}^{n} i F_{4(n-i)}+3 n$ & $(6.7)$ \\
\hline 8. & 4 & 3 & $F_{4 n+1}=5 \sum_{i=1}^{n} i F_{4(n-i)+1}-n+1$ & (6.8) \\
\hline 9. & 4 & $-3,6$ & $F_{4 n+2}=5 \sum_{i=1}^{n} i F_{4(n-i)+2}+2 n+1$ & $(6.9)$ \\
\hline 10. & 4 & 0 & $F_{4 n+3}=5 \sum_{i=1}^{n} i F_{4(n-i)+3}+n+2$. & $(6.10)$ \\
\hline & & & Lucas Identities & \\
\hline 11. & 1 & $1,-4$ & $L_{n}=\sum_{i=1}^{n}(i-2) L_{n-i}+n+2$ & $(6.11)$ \\
\hline 12 & & & $L_{2 n}=\sum_{i=0}^{n-1} i L_{2(n-i)}+n+2$ & $(6.12)$ \\
\hline 13. & 2 & $-3,2$ & $L_{2 n+1}=\sum_{i=1}^{n} i L_{2(n-i)+1}+2 n+1$ & $(6.13)$ \\
\hline 14. & 3 & 5 & $L_{3 n}=2\left\{\sum_{i=1}^{n}(2 i-1) L_{3(n-i)}-n+1\right\}$ & $(6.14)$ \\
\hline 15. & 3 & -5 & $L_{3 n+1}=2 \sum_{i=1}^{n}(2 i-1) L_{3(n-i)+1}+4 n+1$ & $(6.15)$ \\
\hline 16. & 3 & 0,1 & $L_{3 n+2}=2 \sum_{i=1}^{n}(2 i-1) L_{3(n-i)+2}+2 n+3$ & $(6.16)$ \\
\hline 17. & & & $L_{4 n}=5 \sum_{i=1}^{n} i L_{4(n-i)}-5 n+2$ & (6.17) \\
\hline 18. & 4 & 9 & $L_{4 n+1}=5 \sum_{i=1}^{n} i L_{4(n-i)+1}+5 n+1$ & (6.18) \\
\hline 19. & & & $L_{4 n+2}=5 \sum_{i=1}^{n} i L_{4(n-i)+2}+3$ & (6.19) \\
\hline 20. & 4 & -6 & $L_{4 n+3}=5 \sum_{i=1}^{n} i L_{4(n-i)+3}+5 n+4$ & $(6.20)$ \\
\hline
\end{tabular}


The identities are listed in Table 3 in the order of the expressions for $F x$ and then for $L x$ such that the values of $x$ are the positive integers; and then the positive integers of the types: $2 n, 2 n+1,3 n, 3 n+1,3 n+2$, $4 n, 4 n+1,4 n+2$ and $4 n+3$ in the successive rows. The values of $\left(m, S_{0}\right)$ are not found for $(6.7),(6.12),(6.19)$ and (6.20). Applying the rule: $F_{4 n}=F_{4 n+2}-F_{4 n+1}$, we can easily obtain (6.7) from (6.8) and (6.9). In like manner (6.12), (6.17) and (6.19) are the easy consequences of (6.13), (6.18) and (6.20).

\section{(b) Fibonacci and Lucas Identities from Recurrence Relation 2} in Table 4.

Only five alternating signs identities (four Fibonacci and one Lucas) are found by trial. These are listed

\section{Table 4}

\begin{tabular}{|c|c|c|c|c|c|}
\hline Sl. No. & $m$ & $(a, b)$ & $T_{0}$ & Fibonacci and Lucas Identities \\
\hline 1. & 1 & $(3,1)$ & 1 & $F_{n+2}=\sum_{i=1}^{n}(-1)^{i-1}(i+2) F_{n-i+2}+(-1)^{n}$ \\
\hline 2. & 2 & $(5,5)$ & 3 & $F_{2 n+1}=5 \sum_{i=1}^{n}(-1)^{i-1} i F_{2(n-i)+1}+(-1)^{n}(2 n+1)$ \\
\hline 3. & 3 & $(6,4)$ & 2 & $F_{3 n+1}=2 \sum_{i=1}^{n}(-1)^{i-1}(2 i+1) F_{3(n-i)+1}+(-1)^{n}(2 n+1)$ \\
\hline 4. & 4 & $(9,9)$ & 1 & $F_{4 n+2}=9 \sum_{i=1}^{n}(-1)^{i-1} i F_{4(n-i)+2}+(-1)^{n}$ \\
\hline 5. & 1 & $(3,1)$ & 0 & $L_{n+2}=\sum_{i=1}^{n}(-1)^{i-1}(i+2) L_{n-i+2}+(-1)^{n}(2 n+3)$ \\
\hline
\end{tabular}

(c) Generalized Fibonacci Identities from Recurrence Relation 1 and Recurrence Relation 2

(i) From (2.5), we get: $S_{n+2}=S_{n+1}+S_{n}$ for $a=-1$ and $b=1$. On the other hand, the rule for generalized Fibonacci sequence is: $G_{n+1}=G_{n}+G_{n-1}$. Let $S_{1}=G_{0}$ and $S_{2}=G_{1}$. From (2), (2.1), .. (2.5), we then get: $G_{1}=1-G_{0}$ and $S_{n+2}=G_{n+1}$; and finally we get:

For $G_{1}=1-G_{0}$ and $G_{n+1}=G_{n}+G_{n-1}$,

$$
G_{n+1}=\sum_{i=0}^{n-1}(i-1) G_{n-i}+G_{1}+n
$$

(8.1) is a generalized Fibonacci identity with the coefficients in arithmetic progression.

(ii) From (3.5), we get: $T_{n+2}=T_{n+1}+T_{n}$ for $a=3$ and $b=1$. Let $T_{1}=G_{0}, T_{2}=G_{1}$ and $T_{0}=x$. From (3), (3.1), ,.., (3..5), we then get:

For $G_{0}=3 x-4, G_{1}=5 x-7$ and $G_{n+1}=G_{n}+G_{n-1}$,

$$
G_{n+1}=\sum_{i=0}^{n}(-1)^{i}(i+3) G_{n-i}+(-1)^{n+1}\{(n-1) x+4 x-5\}
$$

(8.2) is another generalized Fibonacci identity with the coefficients in arithmetic progression. (8.1) and (8.2) are the same and alternating signs generalized Fibonacci identities respectively.

Remark 1: Periodic Oscillations of the Sequences from Recurrence Relation 1 and Recurrence Relation 2 From (2) and (2.5), we find the sequence: $\left(S_{1}, S_{2}, S_{3}, \ldots\right)$ for $a=b=-1$; and from (3) and (3.5), the sequence: $\left(T_{1}, T_{2}, T_{3}, \ldots\right)$ for $a=b=3$ such that both the sequences have the typical periodic oscillations with the periodic cycles of six elements. The periodic cycle of the first sequence is composed of six elements: $-S_{0}-2,-S_{0}-1,1, S_{0}+2, S_{0}+1,-1$; and the periodic cycle of the second sequence is composed of six elements: $3 T_{0}-6,3 T_{0}-9,-3,6-3 T_{0}, 9-3 T_{0}$ and 3 in succession. The last three elements are the negative values of the first three and vice versa in both the periodic cycles of six elements. Obviously the periodic cycle of the first sequence is independent of the values of $S_{0}$ and the second one is independent of the values of $T_{0}$. It is found by trial that the sequences: $\left(S_{1}, S_{2}, S_{3}, \ldots\right)$ and $\left(T_{1}, T_{2}, T_{3}, \ldots\right)$ can be convergent or divergent depending on the values of the pair $(a, b)$. 


\section{Negative Indexed Fibonacci And Lucas Identities With The Coefficients In Arithmetic Progression}

Negative indexed Fibonacci sequence or extended Fibonacci sequence is : $\ldots F_{-6}, F_{-5}, F_{-4}, F_{-3}, F_{-2}$, $F_{-1}$ defined by the relation: $F_{-n+1}=F_{-n}+F_{-n-1}$ for $n \geq 2$ with the initial conditions: $F_{-1}=1$ and $F_{-2}=-1$. This sequence is also called 'Negafibonacci sequence'. We can obtain Fibonacci sequence with extension: $F s(E)$ by the association of (i) the extended or the left hand sequence: $F s(L)$ and (ii) the usual or the right hand sequence: $F s(R)$. That is, $F s(E)=F s(L)+F s(R)$. Replacing $n$ by $-n$ in the rule for $F s(R)$, we get the rule for $F s(L)$. Fibonacci numbers under $F s(L)$ and $F s(R)$ satisfy the relation: $F_{-n}=(-1)^{n+1} F_{n}$. The special case is: $F_{-0}$ $=F_{0}=0$ for $n=0$, which is under $F s(R) . F_{-0}$ is not a usual symbol. $F s(E)$ is shown in Table 5 .

Table 5

\begin{tabular}{|c|c|c|c|c|c|c|c|c|c|c|c|c|}
\hline \multicolumn{10}{|c|}{ Fibonacci Sequence with Extension: $\boldsymbol{F s}(\boldsymbol{E})$} \\
\hline.. & $\cdots$ & $F_{-17}$ & $F_{-16}$ & $F_{-15}$ & $F_{-14}$ & $F_{-13}$ & $F_{-12}$ & $F_{-11}$ & $F_{-10}$ & $F_{-9}$ & $F_{-8}$ & $F_{-7}$ \\
\hline$\cdots$ & $\cdots$ & 1597 & -987 & 610 & -377 & 233 & -144 & 89 & -55 & 34 & -21 & 13 \\
\hline$F_{-6}$ & $F_{-5}$ & $F_{-4}$ & $F_{-3}$ & $F_{-2}$ & $F_{-1}$ & $\boldsymbol{F}_{\mathbf{0}}$ & $F_{1}$ & $F_{2}$ & $F_{3}$ & $F_{4}$ & $F_{5}$ & $F_{6}$ \\
\hline-8 & 5 & -3 & 2 & -1 & 1 & $\mathbf{0}$ & 1 & 1 & 2 & 3 & 5 & 8 \\
\hline$F_{7}$ & $F_{8}$ & $F_{9}$ & $F_{10}$ & $F_{11}$ & $F_{12}$ & $F_{13}$ & $F_{14}$ & $F_{15}$ & $F_{16}$ & $F_{17}$ & $\cdots$ & $\cdots$ \\
\hline 13 & 21 & 34 & 55 & 89 & 144 & 233 & 377 & 610 & 987 & 1597 & $\cdots$ & $\cdots$ \\
\hline
\end{tabular}

Negative indexed Lucas sequence or extended Lucas sequence is : $\ldots L_{-6}, L_{-5}, L_{-4}, L_{-3}, L_{-2}, L_{-1}$ defined by the relation: $L_{-n+1}=L_{-n}+L_{-n-1}$ for $n \geq 2$ with the initial conditions: $L_{-1}=-1$ and $L_{-2}=3$. Then Lucas sequence with extension: $L s(E)$ is the association of (i) the extended or the left hand sequence: $L s(L)$ and (ii) the usual or the right hand sequence: $L s(R)$. Lucas numbers under $L s(L)$ and $L s(R)$ satisfy the relation: $L_{n}$ $=(-1)^{n+1} L_{-n} . L s(E)$ is shown in Table 6.

Table 6

\begin{tabular}{|c|c|c|c|c|c|c|c|c|c|c|c|c|}
\hline \multicolumn{10}{|c|}{ Lucas Sequence with Extension: $\boldsymbol{L s}(\boldsymbol{E})$} \\
\hline.. & $\ldots$ & $L_{-17}$ & $L_{-16}$ & $L_{-16}$ & $L_{-14}$ & $L_{-13}$ & $L_{-12}$ & $L_{-11}$ & $L_{-10}$ & $L_{-9}$ & $L_{-8}$ & $L_{-7}$ \\
\hline$\ldots$ & $\ldots$ & -1974 & 1354 & -610 & 843 & -521 & 322 & -199 & 123 & -76 & 47 & -29 \\
\hline$L_{-6}$ & $L_{-5}$ & $L_{-4}$ & $L_{-3}$ & $L_{-2}$ & $L_{-1}$ & $\boldsymbol{L}_{0}$ & $L_{1}$ & $L_{2}$ & $L_{3}$ & $L_{4}$ & $L_{5}$ & $L_{6}$ \\
\hline 18 & -11 & 7 & -4 & 3 & -1 & $\mathbf{2}$ & 1 & 3 & 4 & 7 & 11 & 18 \\
\hline$L_{7}$ & $L_{8}$ & $L_{9}$ & $L_{10}$ & $L_{11}$ & $L_{12}$ & $F_{13}$ & $L_{14}$ & $L_{15}$ & $L_{16}$ & $L_{17}$ & $\ldots$ & $\ldots$ \\
\hline 29 & 47 & 76 & 123 & 199 & 322 & 521 & 843 & 1364 & 2207 & 3571 & $\ldots$ & $\ldots$ \\
\hline
\end{tabular}

$F s(E)$ differs from $L s(E)$ remarkably by the following relations:

(i) The numbers under $F s(E)$ satisfy the relations: $F_{2 n}=-F_{-2 n}$ and $F_{2 n-1}=F_{-2 n+1}$.

(ii) The numbers under $L s(E)$ satisfy the relations: $L_{2 n}=L_{-2 n}$ and $L_{2 n-1}=-L_{-2 n+1}$.

Some negative indexed Fibonacci and Luks identities are shown below.

$$
\begin{aligned}
F_{-n-2} & =\sum_{i=2}^{n+1}(-1)^{i+1}(i-2) F_{-n-1+i}+(-1)^{n+1}(n+1) \\
& =-\sum_{i=1}^{n}(i+2) F_{-n-2+i}-1 . \\
F_{-2 n} & =\sum_{i=1}^{n-1} i F_{-2(n-i)}-n .
\end{aligned}
$$




$$
\begin{gathered}
F_{-2 n+1}=\sum_{i=1}^{n-1} i F_{-2(n-i)+1}+1 . \\
F_{-2 n-1}=5 \sum_{i=1}^{n}(-1)^{i-1} i F_{-2(n-i)-1}+(-1)^{n}(2 n+1) . \\
F_{-3 n-1}=-2 \sum_{i=1}^{n}(2 i+1) F_{-3(n-i)-1}+(-1)^{2 n}(2 n+1) . \\
F_{-3 n-2}=2 \sum_{i=1}^{n}(-1)^{i}(2 i-1) F_{-3(n-i)-2}+(-1)^{n+1}(2 n+1) . \\
F_{-4 n+2}=5 \sum_{i=1}^{n-1} i F_{-4(n-i)+2}-2 n+1 \\
F_{-4 n-2}=9 \sum_{i=1}^{n}(-1)^{i-1} i F_{-4(n-i)-2}+(-1)^{n-1} . \\
L_{-n-2}=\sum_{i=2}^{n+1}(-1)^{i+1}(i-2) L_{-n-1+i}+(-1)^{n}(n+3) \\
=-\sum_{i=1}^{n}(i+2) L_{-n+i-2}+2 n+3 . \\
L_{-2 n+1}=\sum_{i=0}^{n-1} i L_{-2}(n-i)+1-2 n+1 .
\end{gathered}
$$

It is curious that the numbers under $F s(E)$ satisfy (1.1) and the numbers under $L s(E)$ satisfy (1.2) for the wide ranges of the values of $n$ and $m$ such that all $n, m \in \mathrm{z}$ with $|n| \geq|m|$.

Examples: Let $|n|=5$ and $|m|=3$. Then the four values of the pair $(n, m)$ are: $(5,3),(5,-3),(-5,3)$ and $(-5,-3)$ which yield four Fibonacci relations from (1.1) and four Lucas relations from (1.2).

(i) From (1.1), the four relations are:

(ii) From (1.2), the four relations are:

$$
\begin{aligned}
& L_{3} F_{5}=F_{8}-F_{2}, \\
& L_{-3} F_{5}=F_{2}-F_{8}, \\
& L_{3} F_{-5}=F_{-2}-F_{-8}, \\
& L_{-3} F_{-5}=F_{-8}-F_{-2}, \\
& L_{3} L_{5}=L_{8}-L_{2} . \\
& L_{-3} L_{5}=L_{2}-L_{8} . \\
& L_{3} L_{-5}=L_{-2}-L_{-8} . \\
& L_{-3} L_{-5}=L_{-8}-L_{-2} .
\end{aligned}
$$

\section{References}

[1]. Arthur Benjamin and Jennifer Quinn, Proofs That Really Count: The Art of Combinatorial Proof, MAA, 2003, pp1-35.

[2]. Bera Soumendra, Relationships between Ordered Compositions and Fibonacci Numbers, Journal of Mathematics Research, CCSE, Vol. 7, No. 3, 2015. Page 4.

[3]. Knuth Donald, Negafibonacci Numbers and the Hyperbolic Plane, http://www.allacademic.com/meta/p206842_index.html

[4]. Steven Vajda, Fibonacci and Lucas Numbers, and the Golden Section: Theory and Applications, Dover Publications, Inc. New York, 2008. 